\title{
Data Body Trader: Identity Augmentation and Post-Biological Organ Trade
}

\author{
Julian Stadon \\ University of the Arts London \\ Salzburg University of Applied Sciences \\ 11D Lewes Crescent, Brighton, UK \\ julianstadon@gmail.com
}

\begin{abstract}
This paper explores the topics of post-biological identity, data bodies and augmentation, particularly through the Data Body Trader project. This research aims to present a speculative, practice based method for understanding post-biological identity, through the establishment of artworks and arts based research endeavours that focus on the relationship between bodies of matter, data bodies and their augmentation in embodied, interactive network scenarios. This paper argues that by having bodies that are both material and virtual, humans are post-biological. This research contributes a framework for understanding post-biological identity that focuses on the mixed reality nature of these ubiquitous, multi-faceted networks of self, through the establishment of the data body as an area of discourse.
\end{abstract}

Data bodies, Mixed reality art. Augmentation. Embodiment. Arts based research. Post-biological identity. Distribution networks. Organ trade. Arts practice.

\section{INTRODUCTION}

The augmentation of data bodies (in other words, data relating to a specific biological identity) has a long history and is in fact, the very foundation of anthropomorphic representation and post-biological identity. This paper will pivot from historical examples of augmentation, past contemporary computer science iterations of the term, towards a more expansive contemporary understanding of the term, that allows for a better understanding of identity and the body. To achieve this, the practical research presents a range of approaches that use post-digital techniques including biometric scanning, mixed reality interfacing and 3D fabrication and post-biological technologies, such as bio-printing, DIY organ farming and open biodata. Through the presentation of artworks, interviews, curation, panel discussions, theoretical discourse and the establishment of a dedicated research organisation, this paper will consolidate a diverse range of outcomes to better define the field and particularly, the author's artistic practice.

\section{BACKGROUND}

The Data Body Trader project commenced as a $\mathrm{PhD}$ research project that continued previous research completed during a Master of Electronic art and has now been a seven-year project. During this time, a deep investigation has been undertaken into the relationship between embodied interaction with art, the body itself, data body creation and distribution and identity. Following several initial theoretical and practice-based research phases, a framework of flexible methods and key terms was established, based around the belief that all bodies and therefore identity, is post-biological. This position was adopted in reference to the augmentation of bodies, in both interactive mixed reality art and data body creation scenarios. (Stadon 2015).

The core goal of this research is to question how art can culturally reconfigure our understanding of post-biological identity, embodiment and data body augmentation and distribution and what methods best facilitate this. To conduct such a transdisciplinary research endeavour, a set of objectives were established that were:

(i) To merge theory relating to mixed reality interfaces, interactive networks, identity and the body within creative art practice.

(ii) To articulate post-biological identity as a result of the representation of augmented data relating to the body in art and science. 
(iii) To define and develop a discourse about data bodies that compares data mining and identity distribution to organ trade.

(iv) To develop a mixed reality organ trade network that is scientifically accurate in its construction and as open source as possible.

(v) To develop a new speculative framework from this, that departs away from traditional binary models of identity and self, towards one more suitable for contemporary ubiquitous networked society, through innovative art practice.

\section{IMPLEMENTATION AND OUTCOMES}

The outcomes of this research offer several original contributions to knowledge, primarily through proposing a speculative practice-based model for exploring post-biological identity, post-digital culture and identity distribution, through using the very techniques (such as digital networks, data body mapping, distribution and fabrication) that have facilitated their emergence (Hansen 2012). To achieve this, certain metaphors such as organ trade are used in juxtaposition with current phenomena such as data mining, hacking and social media, alongside a range of key terms that contextualise this discourse and deliver it in such a way that allows for a continuing development of knowledge in the field. In other words, this research offers a speculative framework that merges cultural theory and practice in a unique method that allows for further growth, due to the open nature of the systems that have been developed (Stadon 2015a).

To best facilitate this in practice, a complex system was developed and presented as an artwork, that through participation, allows both the artist and audience to explore and question their perceptions of and implicitly in emerging phenomena such as data mining and data body creation (defined as information about individual's identity that is linked to their body through biometrics/biodata) (Stadon 2015a). This method of inquiry leads the viewer to question their understandings of ownership, in regards to data bodies, the monetary implications of identity distribution networks (for example social media, ISPs and search engines selling user identified analytical data for targeted adverting, etc.) and what impacts such systems will have on future societies, particularly in the advent of recent developments in postbiological technologies, such as bio-printing, DIY organ farming, bio-genetics and open bio-data.

The Data Body Trader project contributes a unique insight into the relationship between humans, bodies, embodied interaction with digital networks, and contemporary data body creation, aiming to reconfigure our understanding of post biological identity, acknowledging its origins as taking place at the birth of human representation, defined in this research as data body banking (Stadon 2015a).

Beginning with organtrader2010 (Stadon 2015b), the many iterations of this project that evolved have culminated in a complex virtual organ trade network that allows the viewer to 'donate' augmentations of their own viscera as virtual organs (that are in fact clones of open source organ models created using MRI models of the artist's own organs). These organs are placed in a virtual environment with an economy that is monetized rather than simulated (the Second Life Linden Dollar), where the organs can be traded for free or for profit. These organs can then be exported from the virtual trade network and fabricated through 3D printing as substrates for DIY organ farming (and in the future for bioprinting). Such a system has never been developed before and could be considered as a new representational method for expressing postbiological identity and body augmentation.

As mentioned, the outcome of this research consists of several different stages/nodes that are presented as documentation, in workshops, online materials, videos, speculative fictions and DIY instructional guides. The phases of this project are as follows:

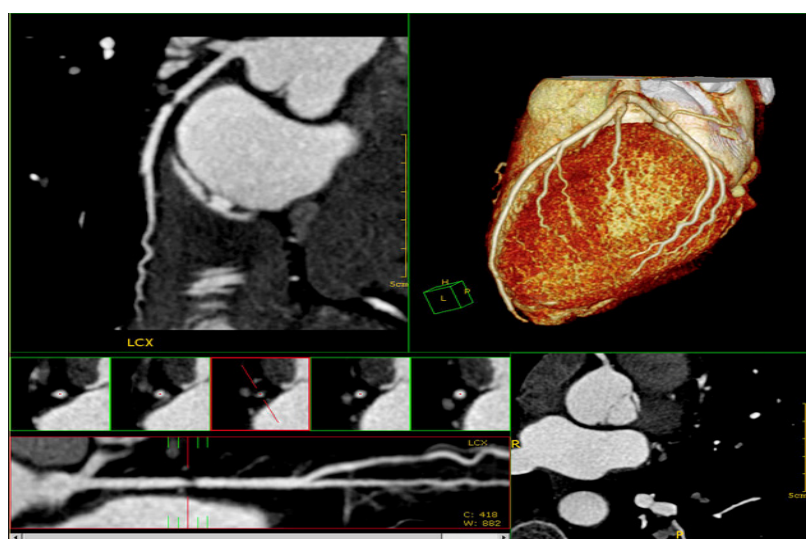

Figure 1: $3 D C T$ Organ scanning.

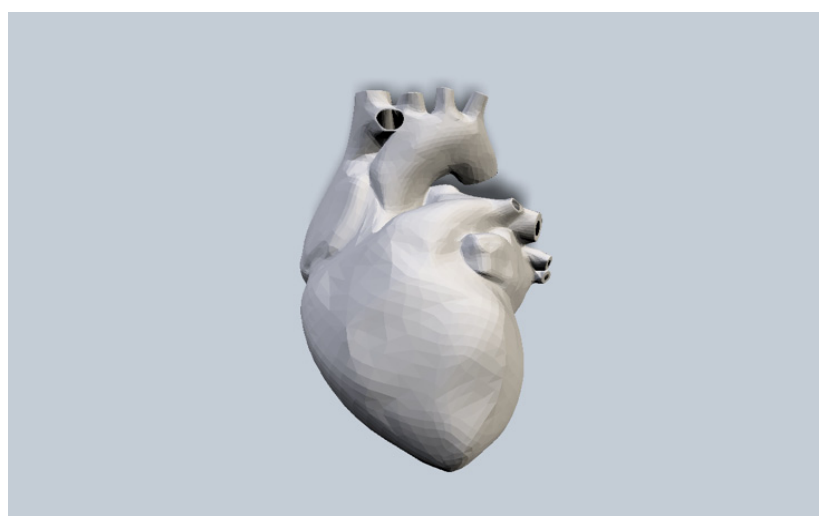

Figure 2: Simplified polygonal model for augmentation and mixed reality data transfer. 


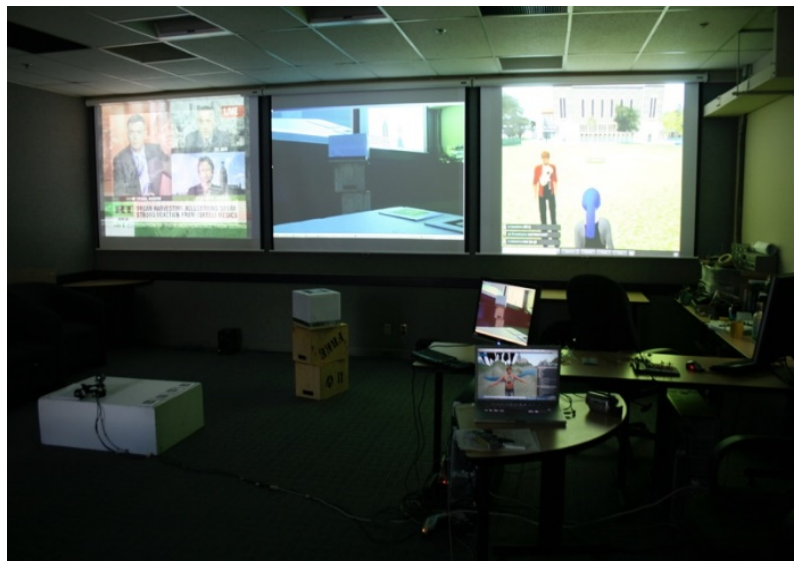

Figure 3: Mixed reality organ trade setup.

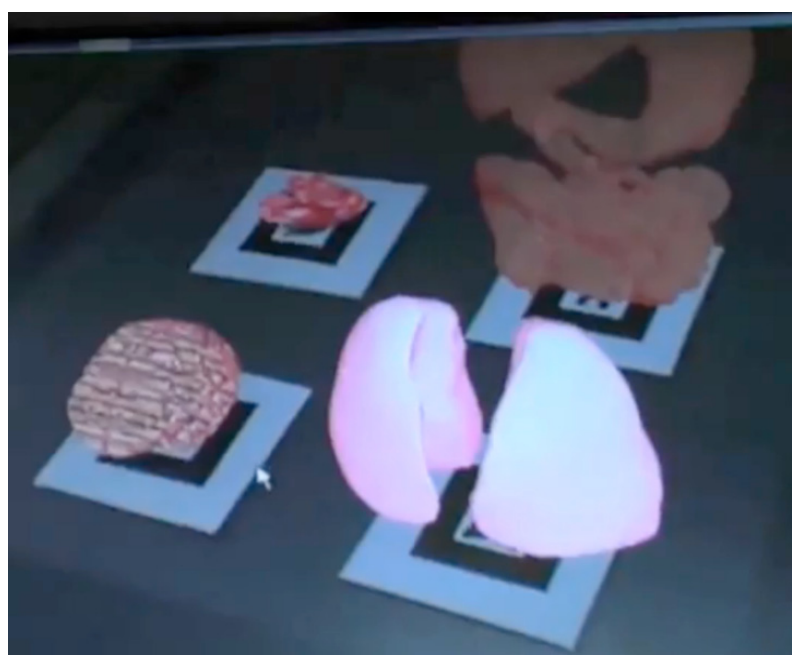

Figure 4: Organ augmentation and mixed reality cloning.

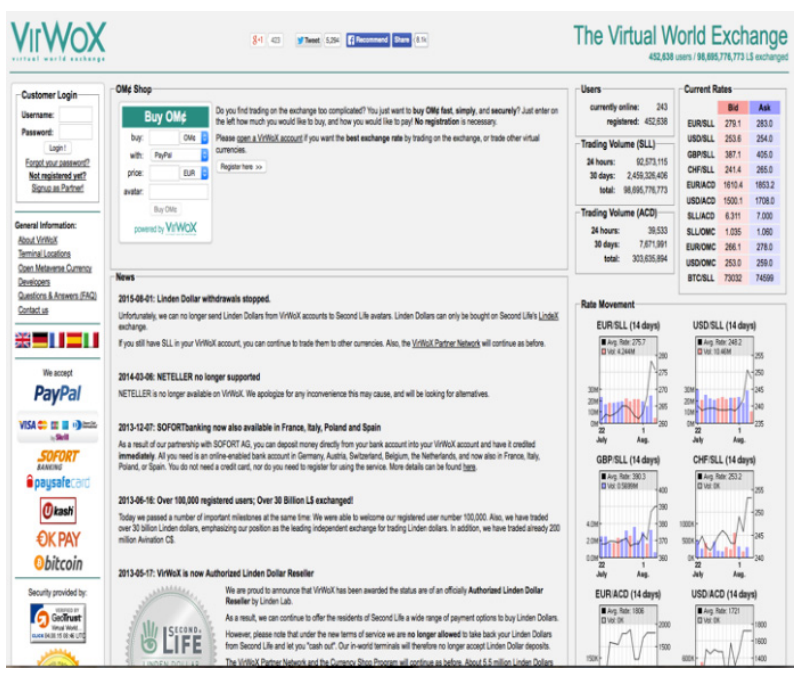

Figure 5: Infiltrating a virtual economy: The Second Life Virtual World Exchange on the US Stock Market.

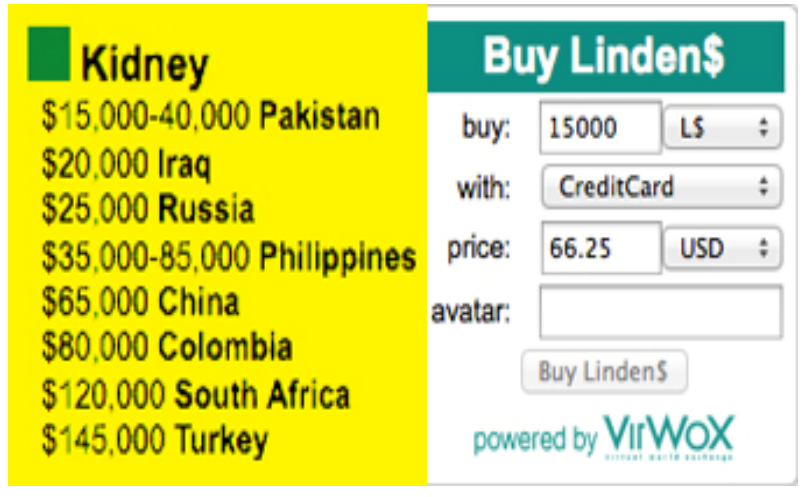

Figure 6: Virtual-Real Price Equivalency Calculation Example

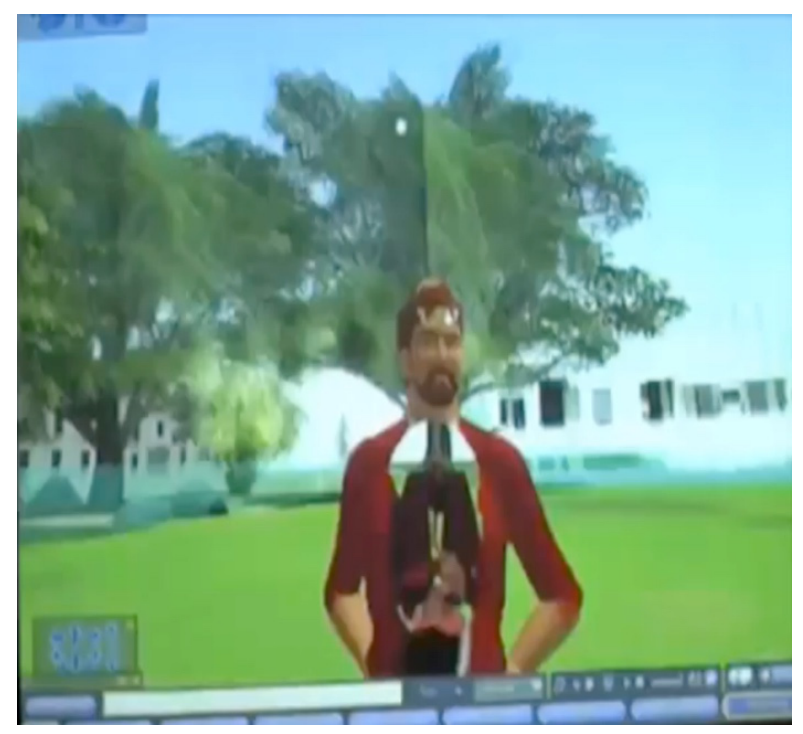

Figure 7: Virtual Avatar/ Organ Trader, equipped with full organ set (worn) ready for trade.

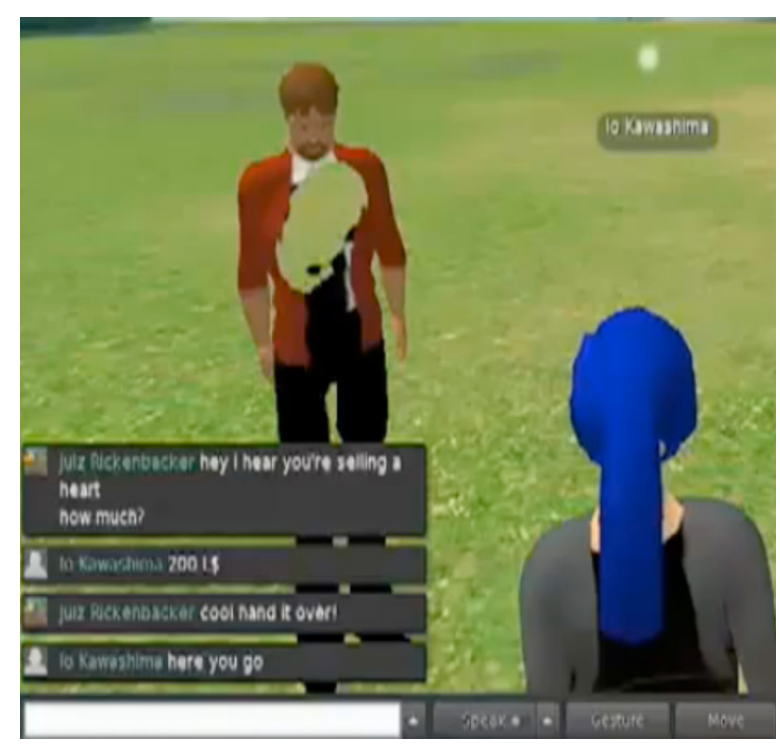

Figure 8: Data body trading, ownership and cloning in a virtual economy. 


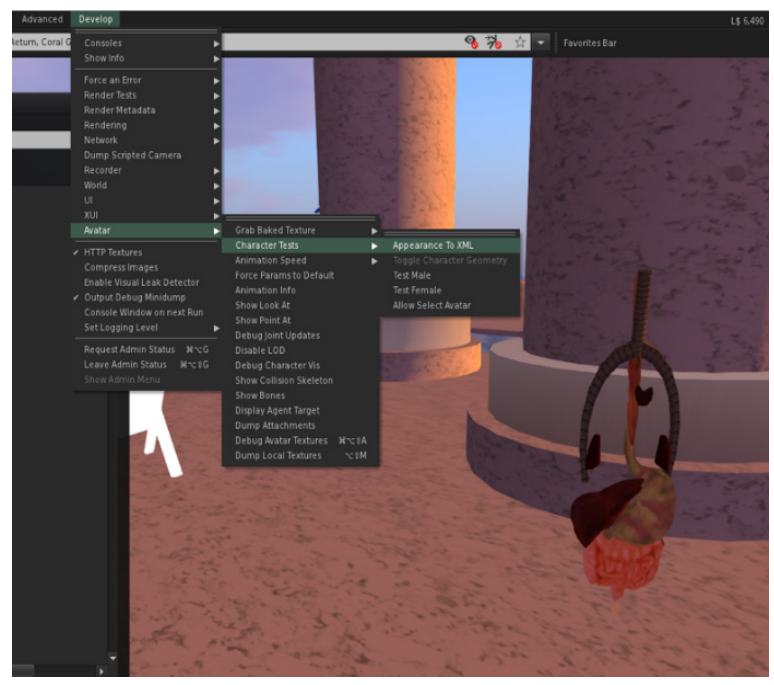

Figure 9: Exporting organs.

\section{LindeX ${ }^{\mathrm{TM}}$ Exchange: Sell L\$}

Just tell us how many Linden dollars you'd like to sell, and the LindeX $X^{\text {Tw }}$ exchange will automatically match you with buyers

Sell Orders may be partially filled: Your Linden Dollars might sell to muttiple buyers, and not all at the same time, and will remain open until theyre sold or you cancel the order. Proceeds are paid at the time of sale, less transaction fees.

For limit sells and detaliled market information, visit the Linden Dollar Exchange Settings for your account and set it to "Advancee". After clicking the "Offer to Sell" button you will be presented with the login page and will need to resubmit your login information to confirm this transaction.

\begin{tabular}{|c|c|c|c|}
\hline Sell Linden Dollars (Market Sell) — & \multicolumn{3}{|c|}{ Your Balances } \\
\hline Quantity of $L S:$ : $649 \mathrm{~d}$ & \multicolumn{2}{|c|}{ L\$6,490 ( } & .000 \\
\hline Estimated Value: $@$ us $\$ 24.73$ & \multicolumn{3}{|c|}{ Your Remaining Lindex Tw Trading Limits } \\
\hline \multirow[t]{3}{*}{ Estimated Proceeds: @ US\$ 23.84} & & per 24 hours & per 30 days \\
\hline & Buy & Us\$1,999.00 & US\$1,999.00 \\
\hline & Sell & US\$1,999.00 & US\$1,999.00 \\
\hline
\end{tabular}

Sell

Figure 10: Banking dividends of data body trade.

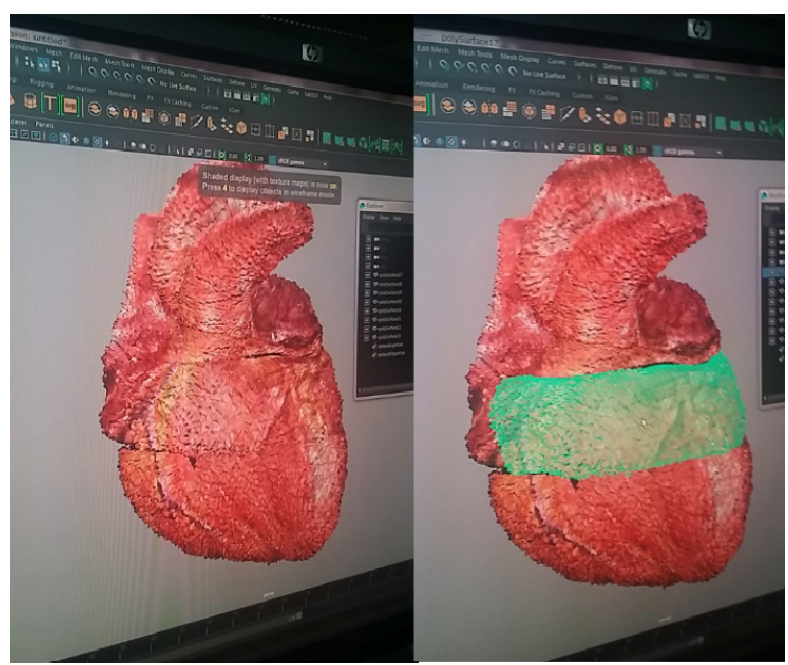

Figure 11: Preparing $3 D$ organ models for $3 D$ printing.

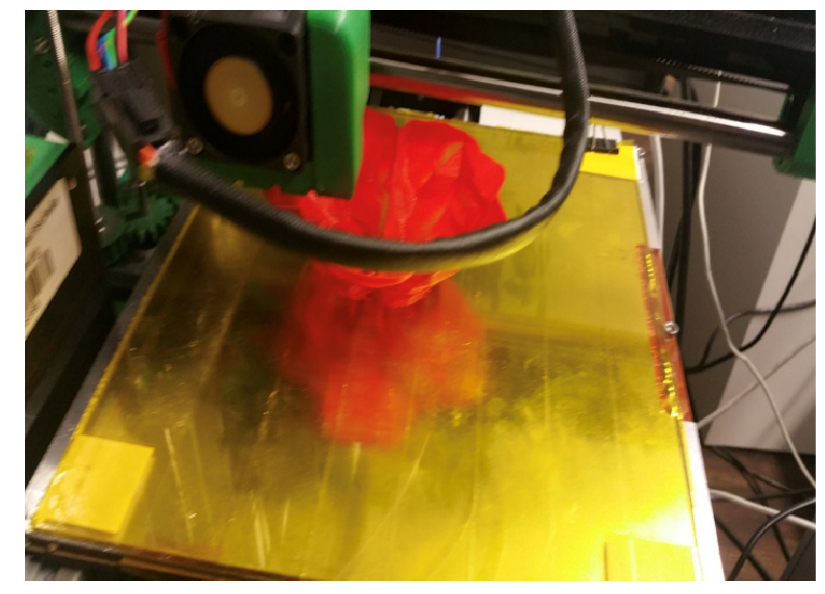

Figure 12: Printing organs as substrates for tissue culture farming.

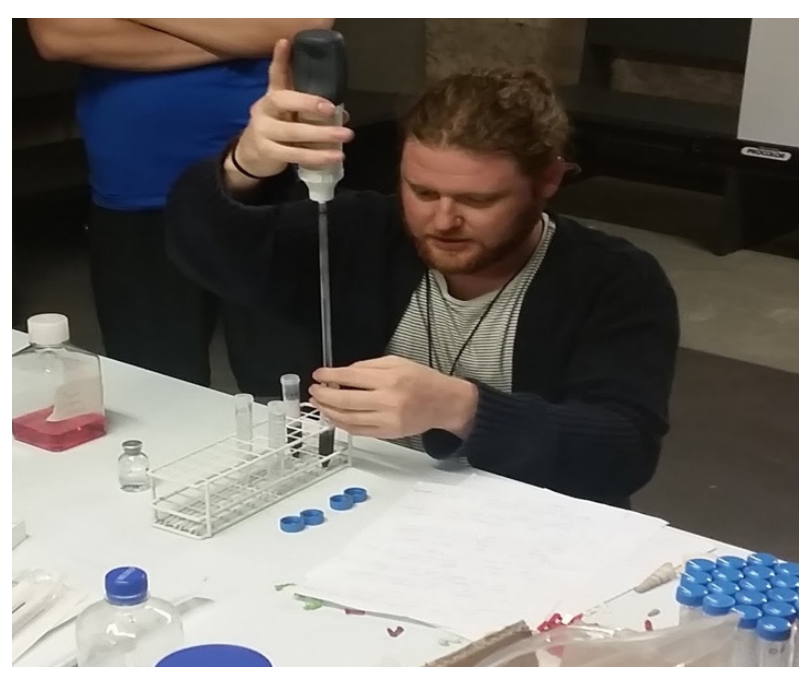

Figure 13: Basic tissue cultivation and DIY organ farming.

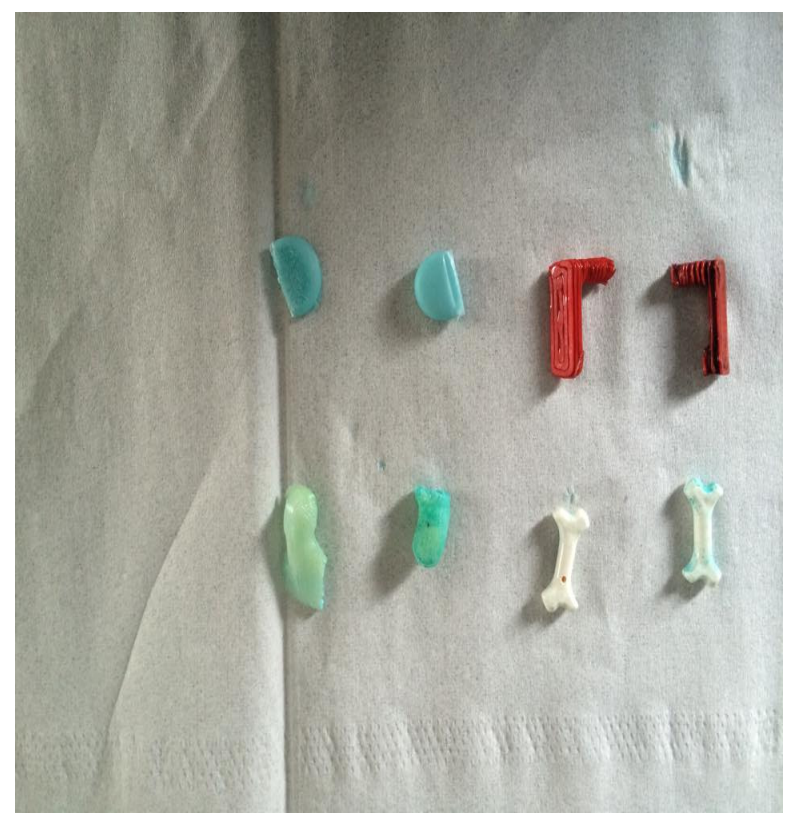

Figure 14: Testing monocyte cell adhesion on different $3 D$ printed materials and substrates. 


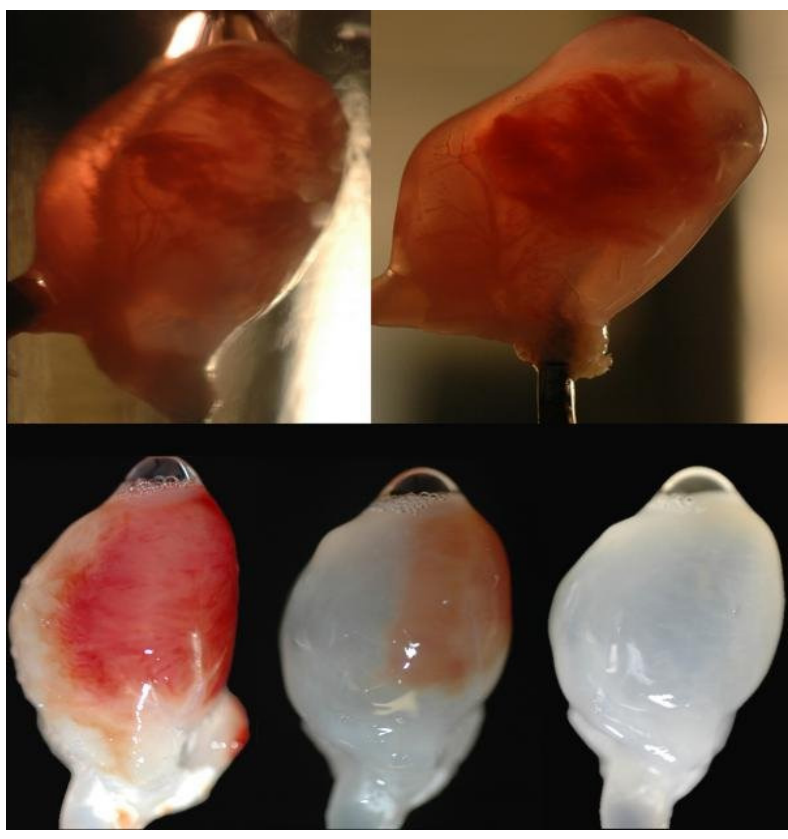

Figure 15: Future research into bio-printing.

It is important to note that this project has been dedicatedly open source and in fact, constitutes a working model for open source organ trade beyond art, in that while utopian in the current context of Bioprinting and tissue culture, it is to some extent, a proven model for organ exchange, cloning and growth: one that has a speculative scientific validity and social value. The written discourse surrounding the practice proposes that such a system could potentially help eradicate organ trade around the world in the future. Though highly speculative, the project is a functioning, open technical network that can is accompanied by DIY instructables, allowing anyone the means to create their own trade network.

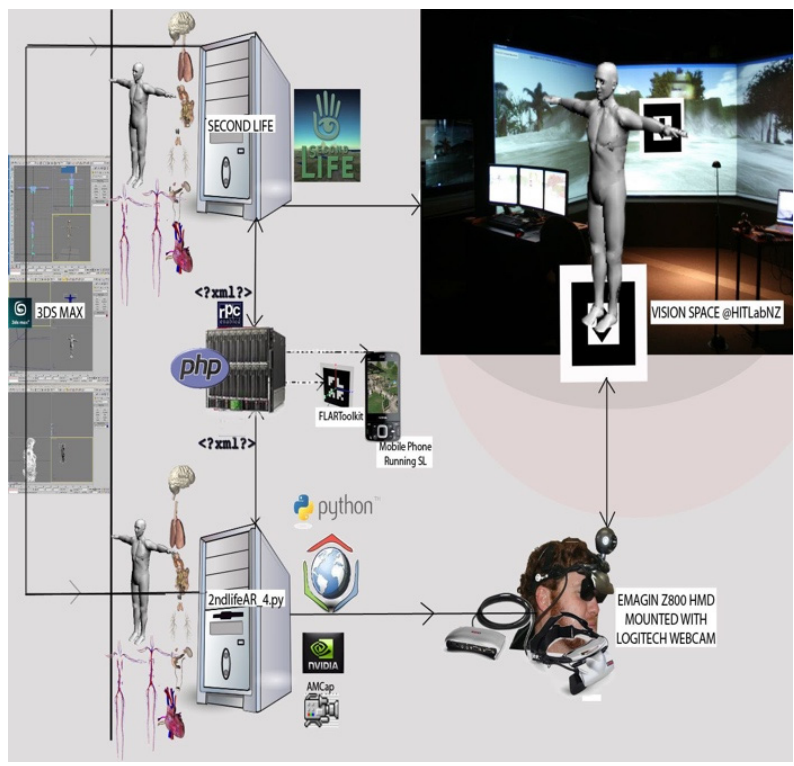

Figure 16: Example of a specifications diagram, including instructions for how to set up the mixed reality data transfer system.

\section{CONCLUSIONS}

By presenting methods of production and proliferation for bodies, as both data and flesh at the same time, this project offers a redefining of common, contemporary understandings of human representation, specifically of our identity and individuality, our bodies and the emergent concept of the data body. Data bodies were historically very permanent, material and objectified, however in this research, they are proven to be atemporal, constantly shifting and evolving and part of a wider economic system.

While originally a passive phenomenon, where representations were primarily dualist (discussed within dichotomies such as virtual/real, I/other, physical/metaphysical and so on), data bodies have now evolved to become symbiotic nondualistic entities for individuals, in relation to both the body and a wider network of biological and post-biological environments. Due to this, it is becoming increasingly necessary that we examine the role of these data bodies, in regards to how we represent them, how they augment us and how we augment them and also how this contributes to the evolution of post-biological identity.

Through a reliance on network integration and distribution, this project decentralizes such dichotomies and allows for a more dispersive approach, consisting of a unique proposition, for a convergence of several points of reference, including Actor Network Theory (Latour 2005), Rhizome (Deleuze and Guattari 1980), Super Fold (Deleuze 1992) and Syncretism (Ascott 2005).

With the monetization of online data, new ethical issues such as ownership and privacy, along with materiality and biopolitics are emerging and as our data body augmentations become more ubiquitous and embedded in our physical/biological bodily existence, the need for serious discourse in this area emerged. This project presents and justifies such a foundation for its discourse, using relevant examples from art and theory to do so (Stadon 2015a).

During this research process, certain gaps have been identified within a range of academic research communities and attempts were made to address these. For example, at the start of this project augmentation was commonly viewed as 'augmented reality', in the wider context of the mixed reality continuum (Milgram et al. 1995), while the concept of augmentation well predates this contemporary scientific understanding of the term. To clarify, upon presentation of this research and observation of other researchers doing similar projects, it was apparent that, perhaps due to it being a rather new field, it was handled too 
generally in art, too specifically in computer science and too vaguely in biological science. Due to this, a reconfiguring of terms was established that aim to specify this ambiguity towards an arts focused discourse. When this was established, an even bigger gap was discovered, that being the generalisation of the field within arts research communities. These terms became the entire foundation for the final consolidation and presentation of this research endeavor and constituted a full chapter in the resulting $\mathrm{PhD}$ thesis, along with a paper specifically dedicated to them, presented at the International Symposium for Electronic Art (ISEA) in 2015 (Stadon 2015a). While these terms, along with the aforementioned flexible points of reference/inspiration based speculative mode of practice were both integral to the success of this project, it was the transdisciplinary approach to interfacing art with a diverse range of both science and humanities fields, that really enabled such a large project for an individual researcher to be achieved.

An important question raised by these statements is: who benefits from this research? To conclude, art surely benefits from a point of view of pushing a higher impact focus on social projects and analysing monetary aspects in regards to the distribution of art and the role artists and the viewer plays in this. Science also can be argued to benefit from such a project, particularly in regards the nature of how such an open transdisciplinary approach can better question the social and ethical implications of emerging technologies and scientific developments, be they from computer science or biotechnology, etc.

Often science labs work in isolated, specific research areas and it is only when these areas are integrated with other fields, that their real impact and potential contribution/damage can be scoped. Questions regarding the body as commodity and the relationship between biological and post biological identity in this context, are becoming increasingly important and in need of further public awareness and discussion. Through its experimental and often hypothetical nature, art provides an opportunity to reconfigure current discourses relating to this and perhaps more importantly, a speculative method for the representation, presentation and public discussion about such a complicated and diverse range of fields and topics.

\section{REFERENCES}

Ascott, R. (2005) Syncretic Reality: Art, Process and Potentiality. Drain, 2.2, online.

Deleuze, G. (1992) Postscript on the Societies of Control. In October, 59, pp. 3-7.

Deleuze, G. and Guattari, F. A. (1980) Thousand Plateaus: Capitalism and Schizophrenia, Vol. 2 of Capitalism and Schizophrenia, 2 vols. 1972-1980. Les Editions de Minuit, Paris, France.

Hansen, M. B. N. (2012) Body Schema as Potentiality. In Hansen, M.B.N (eds.), Bodies in Code: Interfaces with Digital Media. Routledge, New York, US.

Latour, B. (2005) Reassembling the Social: An Introduction to Actor Network Theory. Oxford University Press, Oxford, UK.

Milgram, P., Takemura, H., Utsumi, A., and Koshino, F. (1995) Augmented Reality: A class of displays on the reality-virtuality continuum. In $\mathrm{H}$. Das (ed.), Proc. SPIE 2351, Telemanipulator and Telepresence Technologies, 21 December. DOI: 10.1117/12.197321

Stadon, J. (2015a) Hybrid Ontologies: An Attempt to Define Networked Mixed Reality Art. International Symposium of Electronic Art (ISEA), Vancouver, Canada.

Stadon, J. (2015b) Data Body Banking: Understanding Post-Biological Identity Through Embodied Mixed Reality Art. In K. Ng, J. P. Bowen \& N. Lambert, EVA London 2015: Electronic Visualisation and the Arts. Electronic Workshops in Computing, BCS.

http://ewic.bcs.org/content/ConWebDoc/54906 (accessed 4 May 2017). 